\title{
MODO DE AUTOCONCEPTO EN UNA ADULTA CON DIABETES DURANTE LA PREVEJEZ: UN ESTUDIO DE CASO
}

\section{SELF-CONCEPT MODE IN AN ADULT WITH DIABETES DURING PRE-AGING: A CASE STUDY}

Isaí A. Medina Fernández ${ }^{1}$, Edna I. P. Navarro-Oliva ${ }^{1}$, Aridahi Y. Chi Uitz², Antonio V. Yam Sosa ${ }^{2}$, Ana L. Carrillo Cervantes ${ }^{1}$ y Sandra C. Esparza González'

\author{
${ }^{1}$ Universidad Autónoma de Coahuila. México \\ ${ }^{2}$ Centro Universitario Siglo XXI. México \\ 3Universidad Autónoma de Yucatán. México
}

\section{Abstract}

The condition of being a woman and living with diabetes causes an impact on the adaptation of the disease; Roy's adaptation model points to the self-concept as a psychosocial aspect of how the person is perceived within the environment, trigger responses that can influence in personal and clinical variables. Objective. To describe the adaptation mode of self-concept in a woman in prediabetes stage with type 2 diabetes. Methodology. Single case study with a phenomenological approach. A semistructured interview was conducted; the analysis was through the "Phenomenological Proposal of the Data Obtained in the Interview"; the adaptation level of the self-concept was identified with the instrument VIVEROS03. Results. Participant with 52 years, and 20 years with diabetes. The adaptation level identified was an integrated level. In the process of the disease, there were problems of acceptance, coping, physical affectation and concerns that influence self-concept. The physical self was constituted by the perception and sensation of the body image; the personal self for the spiritual support, ideal self-care, conscious self and the must be. Conclusions. These aspects represent areas of interest and opportunity for nursing, for a humanized approach, integral and with emphasis on psychological aspects.

Keywords: Nursing theory, self concept, diabetes mellitus type 2, adaptation psychological, geriatric nursing.

\section{Resumen}

El ser mujer y vivir con diabetes ocasiona un impacto en la adaptación de la enfermedad; el modelo de adaptación de Roy señala el modo de autoconcepto como aspecto psicosocial de cómo se percibe la persona dentro del ambiente, provocando respuestas que puede influir con determinadas variables personales y clínicas. Objetivo. Describir el modo de adaptación de autoconcepto en una mujer en etapa de prevejez con diabetes tipo 2. Metodología. Estudio de caso único con abordaje fenomenológico. Se realizó una entrevista semistructurada; el análisis fue mediante la "Propuesta Fenomenológico de los Datos Obtenidos en la Entrevista"; el nivel adaptación del autoconcepto (NAA) se identificó con el instrumento VIVEROS03. Resultados. Participante con 52 años, y 20 años viviendo con diabetes. EI NAA identificado fue nivel integrado. En el proceso de la enfermedad se manifestó problemas de aceptación, afrontamiento, afectación física y preocupaciones que influyen en el autoconcepto. El yo físico se constituyó por la percepción y sensación de la imagen corporal; el yo personal por el apoyo espiritual, autocuidado ideal, yo consciente y el deber ser. Conclusiones. Estos aspectos representan áreas de interés y oportunidad para enfermería, para un abordaje humanizado, integral y con énfasis en aspectos psicológico.

Palabras clave: Teoría de Enfermería, autoimagen, diabetes mellitus tipo 2, adaptación psicológica, enfermería geriátrica. 
La etapa de prevejez es un periodo entre los 45 y 59 años, en el cual ocurren cambios psicológicos, sociales y físicos, en el caso de las mujeres es la edad de mayor riesgo para el comienzo de enfermedades crónicas, como la diabetes tipo 2 (DT2) (Chavez, 2011; Servián, 2018). Actualmente se estima que 422 millones (8.5\%) de adultos en todo el mundo presentan diagnóstico de DT2 y en América la prevalencia es del 8.3\% (Organización Mundial de la Salud [OMS], 2016). En México, de acuerdo a la Encuesta Nacional de Salud y Nutrición (ENSANUT) en el 2016 la prevalencia de diabetes fue $9.4 \%$, de los cuales el $25.5 \%$ eran adultos mujeres entre 45 y 59 años (Shamah, Cuevas, Rivera, \& Hernández, 2016). En relación a estos mismos datos, en el estado de Yucatán, las personas con diagnóstico de DT2 fue de 9.2\%, no obstante, la concentración de mujeres con diabetes fue del $11.3 \%$ en etapa de prevejez (Instituto Nacional de Salud Pública [INSP], 2013).

El ser mujer y vivir con diabetes es una condición que impacta directamente en todas las esferas de la persona, por lo cual resulta importante conocer el significado de ser mujer en etapa de prevejez que vive con la enfermedad. Es decir, el significado se ha convertido en piedra angular para la interpretación causal de las enfermedades, de tal forma se considera que la salud y la enfermedad son un conjunto integral de complejos factores biológicos, naturales y sociales (López \& Ávalos, 2013). Así mismo, la diabetes es una enfermedad que tiene su origen en una alteración orgánica, sin embargo, y dada la complejidad de la naturaleza humana, existen variables de orden psicológico que intervienen en el desarrollo de la enfermedad (Torales, Lara, Ruiz, \& Villalba, 2016).

Además, la diabetes puede desarrollar alteraciones psicológicas en particular el autoconcepto, es decir, la percepción que se posee de sí mismo y del mundo que le rodea, el cual tiene un papel relevante en el desarrollo de la vida cotidiana (Serra, 2015). Igualmente, el autoconcepto implica la estabilidad en la autoevaluación a través del tiempo y la coherencia organizacional de sí mismo, en otras pablaras, la autoestima es considerado como un aspecto dominante del autoconcepto y se relaciona con el valor que la persona tiene de sí misma, ya que, a través de una lucha por la autoorganización la persona evita el desequilibrio y el malestar (Roy, 2009).
Así mismo, el autoconcepto es un determinante en el complejo proceso del desarrollo psicosocial de las personas, donde se enmarca la relevancia del mismo para llegar a comprender la forma en que regulan sus propias conductas en diferentes contextos, en consecuencia, se puede encontrar factores que llevan a un autoconcepto específico (Portilla, 2011). Una de las teorías que considera la adaptación psicosocial de los procesos patológicos es el Modelo de Adaptación propuesto por Sor Callista Roy, quien define a la persona como un sistema adaptativo y a su vez a la adaptación como un proceso vital que ocurre como resultado en constante interacción, aunado a ello, propone cuatro formas de comportamiento a los que denomina modos adaptativos y la constituyen el modo fisiológico, modo de interdependencia, modo de función del rol y modo de auto concepto (Roy, 2012).

Roy define al modo de autoconcepto como la necesidad subyacente del individuo, la integridad física, o la necesidad de saber quién es uno, para ser o existir con un sentido de unidad (Roy, 2000). Este modo es caracterizado por la manera en cómo las personas o grupos, se perciben en su entorno dentro del grupo/ambiente donde se desarrollan. Este modo incluye las relaciones interpersonales, autoimagen de grupo, ambiente social, y cultura (Cavalcante, Venícios, \& De Araujo, 2005). Como resultado de la percepción del modo de adaptación existen diferencias en los aspectos psicosociales de los adultos en etapa de prevejez, es decir, depende de un momento determinado en la vida de cada persona, además se considera los diversos rasgos de personalidad y otros condicionantes individuales, aunado a la condición crónica que padecen, en consecuencia puede generar un caos en la cotidianidad de la persona, pudiendo influir en su autoconcepto, por lo que dichos aspectos deben ser considerados en la práctica clínica y comunitaria diaria, para que así sea un aspecto tan relevantes como el tratamiento farmacológico (Cruz, Navarro, Balam, \& Pool, 2017; Mesa, Carrillo, \& Moreno, 2013).

En definitiva, el impacto del diagnóstico, el pronóstico y el tratamiento de la diabetes provoca una serie de respuestas psicológicas y sociales en la persona, que varían en grado e importancia, y que están relacionadas con determinadas variables personales y clínicas (Torales, et al., 2016). De modo que es necesario considerar los aspectos psicosociales de las personas que 
viven con DT2, debido a que deben ser atendidos y entendidos como personas y no como enfermos, ya que pueden ser barreras para comprender mejor porque no logran el automanejo de la enfermedad (López-Ramón, Ávalos-García, Morales-García, \& Priego-Álvarez, 2013). Por lo anteriormente expuesto, se plantea el siguiente objetivo, describir el modo de adaptación de autoconcepto en una mujer que se encuentra en la prevejez y que vive con diabetes tipo 2 (DT2), desde una mirada fenomenológica.

\section{MÉTODO}

\section{Diseño y participantes}

Se realizó un estudio de caso único, al ser relevante comprender a las personas, su experiencia, su percepción y sus emociones (Muñiz, 2010). El abordaje fue fenomenológico descriptivo, ya que el profesional de enfermería centra su interés en lo que experimentan las personas acerca de algún fenómeno, que, a través de la reflexión, se puede descubrir aquello invariable que está presente en las vivencias del ser humano, con el fin de determinar la importancia de los cuidados en las situaciones de vida-salud (Andrea \& Perdomo, 2016).

El estudio se realizó en el domicilio del participante ubicado en la comunidad de Motul, Yucatán, México. Los criterios que se consideraron para la selección el caso fueron: mujer adulta en etapa de prevez, diagnóstico médico de DT2, tiempo de vivir con la enfermedad igual o mayor a 20 años con el fin de identificar si la experiencia de vida desde el modo de autoconcepto varia y que sea originaria de un municipio maya.

\section{Instrumentos}

El nivel de modo de adaptación del autoconcepto se evaluó con el instrumento VIVEROSO3 con un alpha de Cronbach de 85 , consta de 57 ítems que mide todos los componentes del modo de autoconcepto y los clasifica en niveles de adaptación según puntuación obtenida: comprometido entre 57-133 puntos, compensatorio 134209 puntos e integrado 210-285 puntos (Viveros \& Diaz, 2004).

\section{Procedimiento}

Una vez identificada la participante, se procedió a realizar una visita domiciliara, con la finalidad de otorgar información sobre el propósito del estudio, así como los beneficios de su participación en los resultados del estudio, de igual manera se informó sobre la confidencialidad de su participación, ya que en los resultados su nombre fue modificado, así mismo, se mencionó que podía cancelar su participación en cualquier momento, seguido, se inició la lectura del consentimiento informado para su conformidad y firma de participación en el estudio.

Posteriormente, se inició con la entrevista semiestructurada, la cual consistió con una pregunta detonadora: A partir de su diagnóstico de la DT2 ¿cómo se ve a sí mismo físicamente y desde el punto de vista de su apariencia personal?, seguido se abordaron los componentes del modo de autoconcepto como preguntas redireccionadoras. La entrevista fue grabada y se resguardará por un periodo de doce meses.

\section{Ánalisis de datos}

El plan de análisis se basó en la "Propuesta de Análisis Fenomenológico de los Datos Obtenidos en la Entrevista", el cual consiste en la transcripción de la entrevista, seguida de la identificación de categorías de unidades de significado general a partir de lo identificado en la entrevista, posteriormente, se clasificó por colores, seguido de la realización de una matriz para identificar el significado de las categorías y subcategorias, al finalizar el paso anterior, se describió la categoría y subcategorías, así como se demostró los discursos que plasmen el significado, por último, se realizó la verificación de las unidades de significado relevante y conclusión (Marí, Bo, \& Climent, 2010).

El rigor científico se mantuvo por medio de la credibilidad y auditabilidad. La credibilidad hace referencia sobre la recolecta de la información, señala que los argumentos son reconocidos por el informante como una aproximación verdadera a su experiencia vivida, que sean lo más creíble y preciso para la persona; la auditabilidad se logró ya que otro investigador examinó los datos, lo que permitió construir conclusiones similares a las del investigador original en toda la etapa de la recolecta de datos (Castillo \& Vásquez, 2003). Además, se realizó la triangulación de los datos, ya que 
tres investigadores analizaron el mismo fenómeno, por lo tanto, se eliminó el sesgo de sólo contar con el análsis de un único investigador, también se realizó una triangulación teórica a través del Modelo de Adaptación de Callista Roy, para así, contar con una interpretación completa y comprensiva sobre el fenómeno de estudio (Aguilar, Gavira, \& Barroso, 2015).

\section{Consideraciones éticas}

El estudio siguió los aspectos éticos establecidos en el reglamento de la Ley General de Salud en Materia de Investigación para la Salud y en la Norma Oficial Mexicana NOM-012-SSA3-2012 (Secretaria de Salud, 2011), que establece los criterios para la ejecución de proyectos de investigación para la salud en seres humanos, así mismo, se contó con la aprobación del Comité de Ética en Investigación de la Facultad de Enfermería de la Universidad Autónoma de Yucatán, como parte del proyecto de investigación "Adaptación fisiológica y de autoconcepto en personas con diabetes tipo 2 de una comunidad suburbana de Yucatán".

\section{RESULTADOS}

Entre las características biográficas de la participante se señala que al momento de la entrevista tenía 52 años de edad, casada, la familia de tipo extensa, para obtener recursos económicos vende dulces y revistas en el mercado de la comunidad. Entre las características clínicas presenta diagnóstico médico de diabetes tipo 2 desde hace 20 años, peso de 80 kilos y estatura de 1.60 metros, lo que representa un índice de masa corporal de $31.25 \mathrm{~kg} / \mathrm{m} 2$, es decir, obesidad grado uno y una última valoración en ayunas de glucosa en sangre de $156 \mathrm{mg} / \mathrm{dl}$ (la ADA recomienda entre 80-130 $\mathrm{mg} / \mathrm{dl}$ como metas de control) (American Diabetes Association [ADA], 2017).

En cuanto al nivel en el modo de adaptación del autoconcepto, se obtuvo una puntuación de 254 que representa el nivel de adaptación integrado. Al mismo tiempo tuvo una menor puntuación en la autoconcistencia y en el yo ideal; con relación al Análisis Fenomenológico de los Datos que se obtuvieron durante la Entrevista se identificaron 5 categorías y 13 subcategorías, como se muestra en la Tabla 1.
Tabla 1. Modo de adaptación del autoconcepto

\begin{tabular}{ll}
\hline Categoría & Subcategoría \\
\hline 1.Proceso de adaptación de & 1.1 Aceptación de la enfermedad \\
la diabetes tipo 2 & 1.2 Problemas en la adaptación \\
2.Afrontamiento de la & 2.1Afrontamiento ineficaz \\
enfermedad & 2.2 Estrategias de afrontamiento \\
& 2.3 Afrontamiento familiar \\
3.Estímulos que influyen en & 3.1 Afectación física de la diabetes tipo \\
la adaptación & 2 \\
& 3.2 Preocupaciones de la enfermedad \\
4. Yo físico & 4.1 Percepción de imagen corporal \\
& 4.2 Percepción de la sensación \\
& corporal \\
5.Yo personal & 5.1 Apoyo espiritual \\
& 5.2 Autocuidado ideal \\
& 5.3 Yo consciente \\
& 5.4 El deber ser \\
\hline
\end{tabular}

Fuente: Elaboración propia.

\section{Proceso de adaptación de la diabetes tipo 2}

Se identificó que la aceptación de la enfermedad fue complicada, debido a que se negaba asistir a su control glucémico, e incluso postergó durante un año el inicio del autocuidado, al mismo tiempo la entrevistada refirió que con el paso del tiempo se acostumbró y aceptó la enfermedad, siendo evidente, concretamente en el consumo de los medicamentos, comer de manera saludable y a la dificultad de realizar ciertas actividades en su vida diaria.

\section{"[...] Aunque es difícil aceptarlo, pero al pasar el tiempo te vas acostumbrado a que debes tomar tu medicamento a que no debo comer tal cosa y no tomar refresco"}

"[...] Uno debe aceptar su enfermedad y tomar su medicamento para que no suba tu azúcar, porque una vez que se suba y para que se baje es peligroso...eso es para estar estable y seguir viviendo, por eso hay que echarle ganas"

En cuanto a los problemas de la adaptación, el no buscar ayuda para el tratamiento y padecer en sí la enfermedad, son situaciones que posiblemente ocasionan la sensación de sentirse mal emocionalmente, refirió que su vida cambio por las limitaciones alimentarias y el tener que consumir siempre el medicamento; el no adherirse a un tratamiento generó sentimientos negativos como tristeza y por consiguiente la alteración de la glucosa, éste último fue un factor que 
causó la asimilación de la enfermedad e iniciar con el tratamiento.

"[...] Me sentí mal, porque no puedes comer muchas cosas y debes de tomar siempre tu medicamento"

"[...] Pero no quería tomar mi medicamento, tarde en tomarlo me sentaba a llorarlo porque no quería tomar"

\section{Afrontamiento de la enfermedad}

La participante presentó un afrontamiento ineficaz con los cambios de estado de ánimo, lo que quizá ocasionó que no tuviera energía para sobrellevar la enfermedad, de igual manera refirió que no realiza ejercicio y esto repercute en el control glucémico, manifestándose por la hiperglicemia de su última valoración, sin embargo, señaló que se encontraba bien en comparación con las anteriores mediciones, en las que obtuvo valores más elevados de glucosa.

"[...] hay días que no quiero hacer nada, solo tengo ganas de acostarme y dormir, dormir, dormir"

"[...] tengo 156 de azúcar...pero estoy bien porque siempre llegaba a 360, un día llegue a 400 de azúcar"

Así mismo, para sobrellevar la enfermedad la persona realizó estrategias de afrontamiento, en particular la adhesión al tratamiento farmacológico, así como la disminución del consumo de refresco y alimentos con alto contenido calórico, y alcohol; igualmente señaló salir fuera de casa a caminar para no perder fuerza y distraerse, sobre todo cuando se siente triste, y también para respira aire fresco; esto lo realiza con el fin de tratar de vivir y salir adelante con la enfermedad.

"[...] tomo mi medicamento, porque sin eso se también se desanima uno, pero en que se toma uno su medicamento tiene un poco de energía cuando está estable su azúcar"

"[...] Ni modo hay que tratar de vivir, pero hay que tratar de hacer las cosas, si no, no sale uno adelante, pero se tiene que hacer, por más complicado"

Con respecto a la subcategoría afrontamiento familiar le permitió brindar apoyo a la persona en el proceso de la enfermedad, sin embargo, señaló que recibe regaños por algunos de sus hijos por no adherirse al tratamiento establecido, sin embargo, refirió que sigue el tratamiento farmacológico por sus nietos e hijos y la fuerza que necesita la obtiene del apoyo de ellos.

"[...] mis hijos me apoyan en las cosas de la enfermedad, para que no me sienta sola, estamos lográndolo entre mis hijos y mis nietos"

\section{Estímulos que influyen en la adaptación}

La subcategoría afección física de la diabetes tipo 2 influyó en la adaptación, como consecuencia de la enfermedad refiere una limitación en la visión, disminución en el peso sobre todo cuando le diagnosticaron la enfermedad, la presencia de manchas en la cara y cuello. Aunque no todas estas manifestaciones las asocia con el padecimiento.

"[...] Pero antes estaba gordita y ahora empecé a bajar, bajar y bajar y fui con el doctor y me dijeron que era diabético"

"[...] Ya me salieron muchas manchas en mi cara y cuello, pero...no sé si está ligado a la diabetes"

En referencia a la subcategoría preocupaciones de la enfermedad, la inestabilidad en el peso por el temor de que esta situación le lleve a un rebote en los niveles de glucosa. Como resultado de estas situaciones originó que se iniciara con el tratamiento farmacológico porque desde su punto de vista si seguía bajando de peso podría llegar a la muerte y no es sano.

"[...] no me gustaría bajar ni subir, no vaya ser que luego baja o suba vaya tener un rebote en mi azúcar"

"[...] empecé a ver que taba bajaba demasiado... empecé a tomar mi medicamento porque si bajando, bajando, bajando, y dije no eso me iba a llevar a la tumba"

\section{Yo físico}

Por otra parte, se identificó la subcategoría percepción de imagen corporal, en la que la participante se percibe de tez morena, cabello negro, exceso de peso y talla alta, sin embargo, refiere que como resultado de la diabetes modificó su cuerpo, ya que bajó mucho de peso. En su opinión refiere que regresó a su peso ya que controló su enfermedad.

"[...] Me describo como cabello negro, uso lente, piel morena, toy gordita, esten altita" 
"[...] tuve un cambio y si llegué a tener un cambio de bajar demasiado, perdí peso, pero ahora por controlarme ya gané peso"

En la subcategoría percepción de la sensación corporal, refirió sentirse bien con su cuerpo y deseaba permanecer con la misma composición corporal que actualmente tiene. Es importante mencionar que la participante percibe como estabilidad corporal el presentar sobrepeso.

"[...] Me siento bien así, claro...ojala me quede así como mi cuerpo estable"

"[...] Si me gusta cómo me veo ahora, porque me he sentido bien...no me gustaría regresar como de antes...así me quiero quedar, me siento bien con mi peso"

\section{Yo personal}

Acerca, de la categoría Yo personal del modo de adaptación del autoconcepto se identificó la subcategoría apoyo espiritual, de mucho valor para la participante, ya que el creer en un ser supremo le ha permitido salir adelante con su enfermedad, sentirse bien, estar en control, y le facilitó la aceptación de la diabetes, y la adherencia al tratamiento.

"[...] Le pedía a él [dios] que asimile mi enfermedad por favor, poco a poco lo fui asimilando, le fui pidiendo que acepte mi enfermedad"

"[...] Pues le pido mucho a dios...que trate de salir delante de mi enfermedad"

En la subcategoría autocuidado ideal la participante se identificó con las acciones que es capaz de realizar para adaptarse a la enfermedad como el tener un buen control glucémico y mantener un buen estado nutricional, entre las actividades que refirió fueron las siguientes: no consumir refrescos con contenido hipercalóricos, no comer comida sin verduras y con alto contenido de grasas, controlar su carácter y asistir al médico para su control.

"[...] Estoy tratando de no comer tanto refresco y grasa, todo lo que me dice el doctor, para estabilizar mi azúcar y no subir de peso, sino sube más"
"[...] para controlar mi azúcar, debo de no tomar mucho refresco embotellado, no coma grasa, que yo no coma comida seca [comida sin verduras] y que no haga mucho coraje"

Por lo que se refiere a la subcategoría yo consciente la participante refirió tener una personalidad que puede controlar, que la alegría en su vida es diferente al que fue antes del diagnóstico, así mismo, señaló no tener problemas con las personas y que no le afecta lo que digan sus familiares, vecinos y amigos hacia su persona, ella se acepta como es.

"[...] Lo que dice la gente no me interesa...no tengo ningún problema ni con los amigos, vecinos, ni familia...yo me siento bien, así como estoy"

Por último, en la subcategoría el deber ser, la participante refirió ser consciente de las acciones y toma de decisiones correctas para llegar a un buen control de la enfermedad y prevenir complicaciones, así mismo expresa el autocuidado como la adherencia al tratamiento para llegar a esa meta.

"[...] solo depende de mí el cuidarme, como tomar mi medicamento y no como cosas que no debo...yo por ejemplo me cuido mucho, tomo a la hora mi medicamento, apenas tengo un corte me cuido la herida"

\section{DISCUSIÓN}

Con las categorías y subcategorías se develó el fenómeno de estudio, es decir el proceso de adaptación, afrontamiento a la enfermedad, así como los estímulos que influyen en la adaptación de autoconcepto en la participante que vive con DT2 y que se encontraba en etapa de prevejez. El modo de autoconcepto de la persona adulta con diabetes se enfoca en las conductas sobre aspectos psicológicos, espirituales, de sentimientos y creencias que una persona tiene acerca de sí misma en un tiempo determinado (Ayala, 2017).

Con referencia al proceso de adaptación, se constituyó por los problemas que dificultaron este proceso, entre estos el tiempo para su aceptación, es decir el resultado de esta aceptación el apegarse al tratamiento. Estos hallazgos concuerdan con lo que señala Nieto y Prada (2012), en su estudio sobre adultos 
maduros con DT2 después de dos años con la enfermedad, encontraron que logran adaptarse por medio de acciones o respuestas positivas para la aceptación, luego aprender a convivir con ella y la voluntad (Nieto y Prada, 2012). Aceptar la enfermedad es un proceso continuo, debido a que la persona debe ser consciente de que lo vivirá el resto de su vida, por lo que deberá aprender, asumir y llevar a cabo conductas nuevas en su estilo de vida y cambios en la forma de vivir (Epul, 2012).

El afrontamiento ineficaz evidenció el impacto emocional de la enfermedad, el pobre control glucémico y la presencia de cansancio. Lo relevante de esta situación, es la búsqueda y utilización de estrategias para mejorar esta situación, la familia es un recurso que apoya de manera positiva en el control de la enfermedad. Estos hallazgos del estudio son similares a lo reportado por Medina, et al. (2017), donde señala que la emoción negativa presente en las personas con diabetes, como por ejemplo la tristeza, al estar asociado a los efectos por los que cursa la enfermedad como los cambios de humor y el cansancio. Otro estudio señala que el afrontamiento fue negativo frente a la enfermedad debido a que se manifestó tristeza y depresión, sin embargo, por motivaciones internas de la persona, así como el apoyo de la familia y el deseo de seguir adelante, fueron determinantes para el cambio en su visión y en la toma de decisiones para la adherencia al tratamiento (Medina, et al., 2017; Nieto \& Prada, 2012).

El aceptar la enfermedad y el impacto emocional que éste ocasiona en la personas, se relaciona con el subsistema de afrontamiento cognitivo, están enmarcados en la conciencia y tiene relación con los procesos cognoscitivos y emocionales que permiten a la persona interpretar la situación y afrontar los estímulos ambientales con base en el análisis de la situación, fundamentada en su experiencia y educación, y por tanto estos procesos serán sus repuestas o comportamientos (Díaz, Betaconurt, Cheverría, Rodríguez, \& González, 2014). En consecuencia, la adaptación y el afrontamiento están relacionados mutuamente puesto que, para lograr la adaptación de cualquier proceso o enfermedad depende de la manera como la persona afronta la situación que influye en ello, así como múltiples factores como el entorno y procesos propios del ser humano (Nieto \& Prada, 2012). Por otra parte, la participante refirió la presencia de estímulos que dificultan la adaptación de autoconcepto como las preocupaciones a la afectación física de la enfermedad y preocupación en la disminución de peso.

Estos resultados son similares a dos estudios realizados en Yucatán México, los cuales presentaron perdida parcial de la visión, acantosis nigricans, así mismo Yam, Candila, Sierra, y Negrón (2017) refirió en su estudio que la pérdida por las complicaciones de la enfermedad es otra preocupación a la que se enfrentan las personas (Medina, et al., 2016; Yam, et al., 2017). Aunado a lo anterior, Callista Roy refiere que los estímulos pueden ser focales, contextuales y residuales, y están relacionados directamente con los procesos de afrontamiento, y a su vez se relaciona tanto de manera directa como indirecta con los modos de adaptación y que puede influir en la respuesta adaptativa (Villamizar \& Durán, 2012).

En cuanto a la categoría yo físico la participante mencionó que se mira y se siente bien consigo misma, refiriendo que la obesidad la considera como un peso normal y el disminuir de peso es sinónimo de enfermedad. Lo anterior, es similar a lo referido por Nieto y Prada (2012) ya que encontró que los participantes reflejan una opinión positiva sobre su imagen corporal a pesar de presentar cambios físicos como el aumento o disminución de peso, sin embargo, tratan de aceptar su imagen corporal y son conscientes que deben desarrollar un autocuidado para evitar posibles complicaciones (Nieto \& Prada, 2012).

Una posible explicación sobre la percepción corporal de la obesidad como una situación saludable puede ser por la implicación cultural en las comunidades mayas, debido a que las personas consideran la obesidad como un estado de extrema gordura, por lo que no se perciben a sí mismas como obesas, además, por lo tanto, no es considerado como un problema de salud (Asián \& Pasos, 2017). En relación con el yo personal, se encuentra el apoyo espiritual, el cual influye sobre el autocuidado y control glucémico, siendo un facilitador para la adaptación de la enfermedad. Esta situación es similar al referido por Ruiz, Díaz y Martínez (2017), indicando que el sistema de creencias del adulto se respalda en la religión, utilizando actividades religiosas (orar) como alternativa de protección espiritual, que le permite brindar fortaleza emocional y autocontrol (Ruíz, et al., 2017), sin embargo, otro estudio indica que el apoyo espiritual parece ayudar 
a la persona para el control del estado de salud y situaciones futuras de la enfermedad (Medina, et al., 2016).

La participante al referirse del deber ser, se refiere sobre el yo moral-ético, ella es consciente de las acciones que con lleva sus decisiones sobre el control de la enfermedad es por ello que trata de adherirse al tratamiento. Esto difiere con el estudio de Epul (2012) señalando que los participantes evidenciaron una escasa conciencia de enfermedad y responsabilidad ante el mal control de la enfermedad, de igual manera indica que una mayor conciencia de autocuidado les permite adherirse al tratamiento. Tomando en cuenta los dos puntos anteriores, Callista Roy señala como el yo moral, ético y espiritual y son una amplia estructura de creencias y valores, independientemente de la corriente ideológica en la que se crea que le permita establecer entre el deber ser y el quien soy, de igual manera influye en la creencia de un ser supremo como un aspecto fundamental en la vida de muchas personas mayores y que puede contribuir de forma decisiva en su estado de salud (Medina, Polanco, Tello, \& Esparza, 2018), esto indica que la moral-ética-espiritualidad se forja con aquellas relaciones significativas que una persona establece cotidianamente y a lo largo de su vida (Ruíz, et al., 2017).

Al mismo tiempo, la autoconcistencia se observa con la personalidad de la participante, demostrando la capacidad de realizar acciones de autocuidado, así como para afrontar los cambios que tienen en su vida y cuerpo. La literatura señala que la autoconsistencia incluye conductas para mantener una estabilidad emocional y psicológica, como la creación de redes de apoyo, sin embargo, las personas olvidan u omiten estas acciones que ayudan a mantener la armonía del ser, siendo necesaria para mejorar el bienestar y habilidad para afrontar mejor los cambios físicos y psicológicos asociados con la enfermedad (Medina, et al., 2018). Por otra parte, el subcomponente del yo ideal se demostró con la subcategoría del autocuidado ideal, el cual son las acciones capaces para adaptarse a la enfermedad, como un buen control de la enfermedad.

Un estudio refiere que la mejora de la sensación de autocontrol y aumenta su confianza y seguridad, reduce sus miedos y preocupación, ofrece recursos y habilidades para mantener hábitos de vida saludables, facilitando la aceptación de la enfermedad, la autoestima y refuerza la proactividad (Danet, Prieto, Gamboa, Retana, \& March, 2016). Lo anterior hace referencia a las conductas que el individuo realiza para mantener cierto equilibrio $u$ homeostasis en las diferentes esferas del ser, dicho lo anterior, las personas que viven con diabetes conocen y realizan ciertas actividades para mantener un equilibrio (Medina, et al., 2018).

En cuanto a la valoración de la adaptación de autoconcepto indicó un nivel integrado, esto significa que la participante trabaja en las funciones del proceso de vida para resolver las necesidades humanas como el saber quién es uno mismo (Nieto \& Prada, 2012). Un estudio indicó que las personas que tienen diabetes y una autoimagen positiva, tiene auto cuidarse, llevar una vida positiva y controlar la enfermedad (Abdoli, Mardanian, \& Mirzaei, 2012). En conclusión, el presente estudio contribuye a la aplicación del Modelo de Adaptación de Callista Roy a la experiencia vivida desde el modo de autoconcepto en una persona adulta en etapa de prevejez con diabetes tipo 2. Las confirmaciones antes mencionadas forman parte de las respuestas adaptativas de autoconcepto, las cuales son útiles para el manejo de la enfermedad, integrando la experiencia de la enfermedad crónica con el concepto de sí mismo y la identidad personal (Luengo \& Salazar, 2008).

No obstante, el otorgar un significado a la adaptación de autoconcepto, ha permitido describir y comprender que está determinado por sus experiencias vividas con la enfermedad. Se identificó el proceso de adaptación mediante la aceptación y presencia de problemas para la participante, de igual manera el afrontamiento hacia la enfermedad es ineficaz, sin embargo, cuenta con estrategias para afrontarlo. Por otra parte, hubo la presencia de estímulos que influyen en la adaptación como la afección física y preocupaciones de lo que podría ocasionar la enfermedad, Roy lo llama estímulos ambientales que influye en el modo de adaptación autoconcepto de manera directa e indirecta.

En relación con el modo de autoconcepto, se observó con el yo físico mediante la aceptación de la imagen corporal y sentirse bien consigo misma, sin embargo, el factor cultural influyó en la percepción a considerar desear quedarse con obesidad, debido que el bajo de peso lo considera como sinónimo de enfermedad. Así mismo el yo personal se identificó mediante el apoyo espiritual que facilita el autocuidado y 
aceptación de la enfermedad, el autocuidado ideal son las acciones capaces para adaptar la enfermedad como el tener un buen control glucémico y adherencia al tratamiento farmacológico y no farmacológico; lo anterior es con ayuda del yo consciente que es la personalidad, indicando que no le afecta en el autoconcepto lo que dicen los demás y por último el aspecto ético-moral está plasmado en la subcategoría del deber ser, la participante sabe de las acciones y toma decisiones adecuadas para su cuidado.

La importancia de esta investigación en atención en salud permitirá mejorar el manejo de la enfermedad por parte del profesional de enfermería con un lado humanizado, incluyendo un abordaje integral, haciendo énfasis el aspecto psicológico, específicamente del autoconcepto de las personas con alguna enfermedad diabetes tipo 2. De igual manera saber la realidad permitirá desarrollar futuras intervenciones que mejoren la adaptación de la persona quien atraviesa por un proceso largo y aprender a convivir con la enfermedad y consigo misma.

\section{REFERENCIAS}

Abdoli, S., Mardanian, L., \& Mirzaei, M. (2012). How public perceive diabetes: A qualitative study. Iranian Journal of Nursing and Midwifery Research, 17(5), 370-374.

Aguilar Gavira, S., \& Barroso Osuna, J. (2015). La triangulación de datos como estrategia en investigación educativa. Píxel-Bit, Revista de Medios y Educación, (47), 73-88. doi: 10.12795/pixelbit.2015.i47.05

American Diabetes Association. (2017). Glycemic targets. Diabetes Care, 40(1), 548-556.

Andrea, C., \& Perdomo, R. (2016). Fenomenología hermenéutica y sus implicaciones en enfermería. Index Enfermería, 25(12), 82-85.

Asián, R., \& Pasos, R. (2017). Sobrepeso y Obesidad en Comunidades Indígenas Mayas. Economía, Cultura y Género. Revista de Estudios Regionales, (109), 139-163.

Ayala, G. (2017). Programa de adaptación del adulto mayor con diabetes mellitus tipo2, aplicando la teoría de Callista Roy en el Hospital de Ate Vitarte- 2014. (Tesis de pregrado). Universidad Alas Peruanas. Perú.

Castillo, E., \& Vásquez, M. L. (2003). El rigor metodológico en la investigación cualitativa. Colombia Médica, 34(3), 164-167. doi: $10.25100 / \mathrm{cm} . v 48 \mathrm{i} 4.3662$

Cavalcante, M., Venícios, M., \& De Araujo, T. (2005). Estudio de la evidencia del concepto de grupo en el modelo de adaptación de Roy. Cultura de Los Cuidados, 9(17), 82-87.
Chavez, A. (2011). Duelo y depresión en el adulto mayor. Asociación Mexicana de Tanatología.

Cruz, A., Navarro, M., Balam, M., \& Pool, R. (2017). Efecto de la motivación conductual en el nivel de adaptación del modo autoconcepto del adulto mayor con hipertensión. Revista Salud y Bienestar Social, 1(1), 25-53.

Danet, A., Prieto, M., Gamboa, E., Retana, L., \& March, J. (2016). La formación entre iguales para pacientes con diabetes mellitus 2. Una evaluación cuantitativa y cualitativa en el País Vasco y Andalucía. Atención Primaria, 48(8), 507-517. doi: 0.1016/j.aprim.2015.10.010

Díaz, A., Betaconurt, C., Cheverría, S., Rodríguez, E., \& González, C. (2014). Diabetes Mellitus: un estudio sobre el nivel de adaptación fisiológica y de autoconcepto en pacientes que participan en un grupo de apoyo. Revista Iberoamericana de Producción Académica Y Gestión Educativa, 1, 1-28.

Epul, P. (2012). Factores que influyen en el manejo de la diabetes tipo I/ en el paciente bajo control del consultorio adosados al hospital San José de Maipo en el año 2010 (tesis de pregrado). Universidad de Chile. Chile

Instituto Nacional de Salud Pública. (2013). Encuesta Nacional de Salud y Nutrición 2012. Resultados por entidad federativa, Yucatán. México.

López-Ramón, C., Ávalos-García, M. I., Morales-García, M., \& PriegoÁlvarez, H. (2013). Perspectivas del paciente en el control de la diabetes mellitus en unidades médicas de la seguridad social de Tabasco Un estudio cualitativo. Salud En Tabasco, 19(3), 77-84.

López, C., \& Ávalos, M. (2013). Diabetes Mellitus tipo 2: barreras y perspectivas en el control del paciente. Horizonte Sanitario, 12(2), 63-69.

Luengo, S., \& Salazar, A. (2008). Aplicación del proceso de enfermería según el Modelo de Roy en pacientes con Diabetes Mellitus. Enfermería Globlal, 14, 1-13.

Marí, R., Bo, R., \& Climent, C. (2010). Propuesta de Análisis Fenomenológico de los Datos Obtenidos en la Entrevista. UT. Revista de Ciències de l'Educació, 113-132. doi: $\underline{10.17345 / \text { ute. } 2010.1 .643}$

Medina, I., Cervera, E., Polanco, T., Tun, M., Cob, A., Salazar, R., \& Candila, J. (2016). Experiencia social de vivir con diabetes tipo 2: una mirada de una comunidad suburbana. (pp. 556-564). Saltillo In Ciencia y humanidades.

Medina, I., Medina, J., Candila, J., Yam, A., Ceballos, J., \& Zavala, D. (2017) Mi vida antes y después del diagnóstico de la diabetes tipo 2. Revista Salud y Bienestar Social, 1(1), 94-114.

Medina, I., Polanco, P., Tello, M., \& Esparza, S. (2018). Propuesta de cuidados de enfermería en la adaptación de autoconcepto de adultos mayores con diabetes tipo 2. Revista Española de Salud Mental, 6, 27-32.

Mesa, L., Carrillo, A., \& Moreno, F. (2013). La cronicidad y sus matices: estudio documental. Investigación En Enfermería: Imagen y Desarrollo, 15(2), 95-114.

Muñiz, M. (2010). Estudios de caso en la investigación cualitativa. División de Estudios de Posgrado. Recuperado de: 
https://psico.edu.uy/sites/default/files/cursos/1 estudios-de-casoen-la-investigacion-cualitativa.pdf

Nieto, C., \& Prada, Á. (2012). Proceso de adaptación de los adultos maduros con diabetes mellitus tipo II atendidos en el H.N.A.A.A Chiclayo-2011 (tesis de pregrado). Universidad Católica Santo Toribio De Mongrovejo. Perú.

Organización Mundial de la Salud. (2016). Informe Mundial sobre la diabetes. Resumen de orientación. doi: 10.18004/rvspmi/2312$\underline{3893 / 2016.03(02) 71-076}$

Portilla, L. (2011). Calidad de vida y autoconcepto en pacientes con diabetes con y sin adherencia al tratamiento (tesis de pregrado). Universidad Mayor de San Marcos. Perú.

Roy, C. (2012). El modelo de adaptación de Roy en el contexto de los modelos de enfermería, con ejemplos de aplicaciones y dificultades. Cultura de Los Cuidados, IV(7/8), 139-159.

Roy, C. (2009). The Roy Adaptation Model. Appleton \& Lange, Ed. Standford.

Ruíz, N., Díaz, A., \& Martínez, J. (2017). Modo adaptativo de autoconcepto : un acercamiento con la experiencia vivida del adulto mayor. Enfermería Comunitaria, 13, 1-7.

Secretaría de Salud (2011). Que Establece los Criterios para la Ejecución de Proyectos de Investigación para la Salud en Seres Humanos.

Serra, A., (2015). Lo que un buen autoconcepto puede hacer por nosotros. C. Revista Iberoamericana Psicopática, 116, 47-56.

Servián, R. (2018). Diabetes y mujer en las distintas etapas de la vida. Recuperado de: https://diabetesmadrid.org/diabetes-y-mujeretapas-de-la-vida/\#Mujeres_mayores de 45_anos

Shamah, T., Cuevas, L., Rivera, J., \& Hernández, M. (2016). Encuesta Nacional de Salud y Nutrición de Medio Camino 2016. doi: $\underline{10.21149 / 8593}$

Torales, J., Lara, G., Ruiz, C., \& Villalba, J. (2016). Aspectos psicopatológicos en el paciente con diabetes. (pp. 16-25).Paraguay. Pie diabético: Manual de manejo.

Villamizar, B., \& Durán, M. (2012). Modelo de adaptación de Roy en un ensayo clínico controlado. Avances En Enfermería, XXX(2), 97-107.

Viveros, M., \& Diaz, R. (2004). Instrumento Viveros 03 para Medir el Autoconcepto de Personas con Enfermedades Crónicas. Desarrollo Científico de Enfermería, 12(4), 113-117.

Yam, A., Candila, J., Sierra, A., \& Negrón, J. (2017). Preocupaciones y temores como experiencia de vivir con diabetes tipo 2. Revista Salud y Bienestar Social, 1(2), 47-61. 